\title{
Reasoning about Potential Hidden Traffic Participants by Tracking Occluded Areas
}

\author{
Lingguang Wang, Christoph Burger and Christoph Stiller
}

\begin{abstract}
Autonomous vehicles face big challenges guaranteeing provable safety during driving. One of the major problems is the uncertainty arising from the perception of the surrounding environment, especially due to occlusions. Recent approaches to tackle these uncertainties either minimize collision risk probabilistically or assume worst-case vehicles coming out of occluded areas. The former does not provide any safety guarantees, while the latter tends to produce overly conservative driving behavior. Human drivers, however, can reason about possible traffic participants (TPs) in occlusions with the knowledge about the street, more importantly, with the continuous observation on the changes of the field of view when moving forward. In this paper, we present an approach that imitates this human-like intelligence and can reason about all the potential TPs in occlusions. By moving forward and propagating the initial knowledge with new observations, the approach can refine the possible states of the TPs in occlusions instead of always adopting a worst-case assumption. By planning w.r.t. the set-based occupancy prediction from the refined state intervals, the vehicle can drive more efficiently under occlusions while guaranteeing safety under reasonable assumptions. The proposed method is evaluated with numerical experiments showing that all the possible hidden TPs can be covered by our refined state intervals, and with that achieving significantly more driving efficiency under occlusions while being safe.
\end{abstract}

\section{INTRODUCTION}

Autonomous driving has received great attention from many research institutions. However, it demands large effort in figuring out how to equip autonomous vehicles with human-like intelligence. In the last years, great progress has been made in the field of sensors and other onboard hardware. Thanks to the advances in machine learning technology, the perception algorithms have improved significantly in recent years as well [1]. However, due to the physical limitation, all the sensors have a maximum sensing range. And more importantly, there will be various surrounding obstacles that block the sensor's field-of-view (FoV), and therefore formulate occlusions, e.g. for the scene shown in Fig. 1(a). As there might be TPs that have right of way over us in the occlusions, the ego vehicle should take them into account, and generate trajectories that can tackle the potential hidden TPs appropriately.

According to the concept of Responsibility Sensitive Safety (RSS) [2], the right-of-way should be given but not taken. Therefore, all the possible states of the TPs in the occluded right-of-way area should be considered. One

Lingguang Wang, Christoph Burger and Christoph Stiller are with the Institute of Measurement and Control Systems, Karlsruhe Institute of Technology (KIT), Karlsruhe, Germany lingguang.wang, christoph.burger, stiller@kit.edu

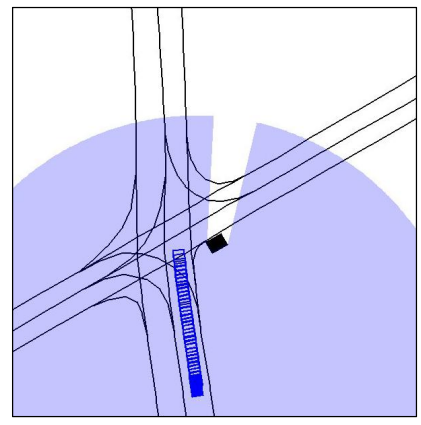

(a)

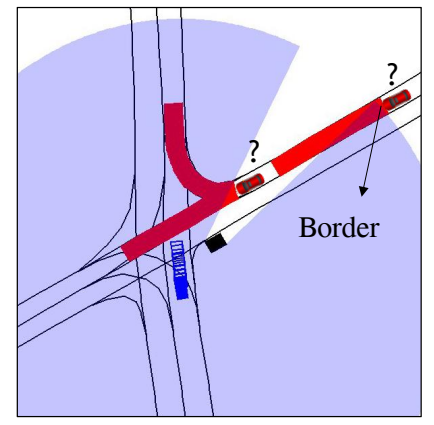

(b)
Fig. 1: Traversing one unsignalized intersection with static occlusion. (a) Ego vehicle (blue solid rectangle) approaches intersection following its trajectory (blue hollow rectangles). (b) Ego vehicle slows down because of the over-approximated reachable sets prediction (red polygons) of the possible hidden TPs.

previous work over-approximate the possible TPs' states in occlusions by state intervals [3]. As depicted in Fig. 1(b), when the ego vehicle approaches one intersection with one obstacle that blocks the FoV of the ego vehicle on the right arm of the street, [3] assumes that there will be vehicles coming out from the sensing edge and sensing border with velocity up to the speed limit at any time. To guarantee safety, it extends the set-based prediction method [4] to the state intervals of the possible TPs to provide occupancy predictions, which are shown by the red polygons. As long as the trajectory of the ego vehicle does not intersect with those reachable sets, safety is guaranteed. However, this assumption can lead to overly conservative driving behavior.

At the position shown in Fig. 1(b), human drivers would not drive only according to the current, but also past evolution of the scene. If they came from the position depicted in Fig. 1(a), where they already had a view on different parts of the street, they would not reason about TPs coming from occlusions with up to maximum velocity, but with lower velocity. Especially, if their FoV can cover the whole street during the time from Fig. 1(a) to Fig. 1(b), and they keep track on the border of the street that no new vehicle enters the occlusion, they can even know that the street is clear, under the mild assumption that no vehicle in the occlusion drives backward.

Motivated by that, this paper proposes an algorithm that can track the occlusions over time, and reason about the states of the possible hidden TPs. We first initialize overapproximated state intervals the first time we observe occlusions on the interesting street, as [3] do. By propagating 
the initialized state intervals and updating them with new observations, they will be gradually refined to the real possible state intervals. With this information, the ego vehicle can plan a trajectory that is not only provably safe in the planning horizon, but also significantly more efficient.

The rest of the paper is organized as follows: Section II introduces the related work. In Section III we explain our approach in detail. Then the evaluations are presented in Section IV. Finally, we conclude this paper and discuss future work in Section V.

\section{RELATED WORK}

Previous works try to tackle occlusions in different ways. One focus is to provide provable safety in scenarios with occlusions. As mentioned before, [3] always assumes worstcase situation when encountering occlusions to provide provable safety. The authors also extend the approach towards RSS by [5] to formalize dangerous situations and proper responses. The authors of [6] maintain the same assumption as [3]. However, they choose a more efficient and comfortable plan at intersections where the probability of hidden TPs is very low according to the traffic flow data. Safety is still guaranteed, but if the probability of a hidden vehicle is small, they prefer one harsh braking instead of 100 unnecessary soft brakings when crossing an intersection 100 times.

Another direction of research focuses on estimating collision risk caused by the potential hidden TPs in occlusions, e.g. [7], [8] and [9]. [7] obtains a comfort plan by utilizing an optimization-based motion planner to reduce the risk, while [8] evaluates the driver's current behavior in terms of risk, warns the driver in case of critical events. As they regard risk as soft constraints, safety guarantee is not provided.

Recently, [10] and [11] formulate the planning problem as Partially Observable Markov Decision Process (POMDP). They predict the future FoV of the autonomous car over the whole planning horizon and achieve a human-like driving behavior when facing occlusions. [12] proposes to learn a driving policy at unsignalized occluded intersections via reinforcement learning, which considers the future occlusions implicitly by maximizing future reward. Other approaches like [13] and [14] incorporate active exploration to eliminate future occlusion as much as possible, to actively reduce the possibility of potential hidden TPs.

However, all of those approaches neglect the historical information about the occlusions that can be used to reason about the current hidden TPs.

Our main contributions adressed in this work are:

- By tracking and propagating the scene with new observations over time, we can refine the state intervals of the potential TPs in occlusions, instead of assuming worst-case situation all the time.

- With the refined state intervals that still cover all the current possible TPs' states in occlusions, a provably safe and more efficient driving behavior is achieved.

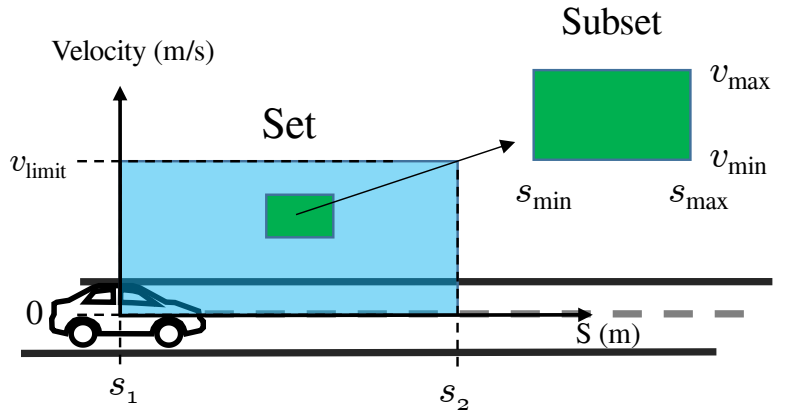

Fig. 2: Definition of set and subset. Along the street, the longitudinal position of the vehicle can be from $s_{1}$ to $s_{2}$. The velocity of the vehicle can be between $0 \frac{\mathrm{m}}{\mathrm{s}}$ to $v_{\text {limit }}$. The state set $\mathcal{S}$ is shown with the transparent blue rectangle. One subset example $\mathbf{S}$ is depicted by the green rectangle.

\section{APPROACH}

In Section III-A, instead of using the term of state intervals, we introduce our definition of state set and subsets. We define three operations on the subsets in Section III$\mathrm{B}$, in order to predict and update the subsets over time. In Section III-C, we will explain the whole pipeline of tracking the subsets with the operations in Section III-B. The resulting subsets can be used to generate reachable set prediction from the occlusions for the behavior planning algorithms.

\section{A. Definition of state set and subset}

In this paper, we introduce two mild assumptions. Firstly, the vehicles are assumed to move along the centerline of the lane, and the lateral offset to the centerline is neglected. The reason is that, from occlusions, what affects the driving behavior the most are the longitudinal position and velocity of the potential vehicles relative to the collision zone, but their lateral position w.r.t. to the centerline is less of interest. However, the concept that will be introduced in the following sections are applicable for considering more dimensions like lateral position, orientation as well, in order to model more TPs like pedestrians and cyclists. This could be an interesting future work that is discussed in Section V. Knowing the longitudinal position w.r.t. the centerline of the lane, the global coordinates of the vehicle can be retrieved by utilizing the High-Definition Map frameworks, e.g. Lanelet2 [15], which models the geometry of a lane with consecutive lanelets. The second mild assumption is, no vehicle drives backward, i.e. with velocity lower than $0 \frac{\mathrm{m}}{\mathrm{s}}$.

Therefore, the state of a vehicle is represented as: the longitudinal position and the velocity. Fig. 2 shows one example. The state set of the vehicle is then a two-dimensional space defined by $\mathcal{S}=\{(s, v)\}$ with $s \in\left[s_{1}, s_{2}\right]$ and $v \in\left[0 \frac{\mathrm{m}}{\mathrm{s}}, v_{\text {limit }}\right]$. One subset $\mathbf{S}$ can be arbitrary part of the set $\mathbf{S} \in \mathcal{S}$, which can be represented by $\mathbf{S}=\{(s, v)\}$ with $s \in\left[s_{\min }, s_{\max }\right]$ and $v \in\left[v_{\min }, v_{\max }\right]$. Each subset $\mathbf{S}$ contains a part of possible states of vehicles on the lane.

When the target lane is occluded, we can over-approximate all the possible vehicles' states in the occlusions by creating several sets $\left[\mathcal{S}_{1}, \mathcal{S}_{2}, \ldots\right]$ on each of the occluded sections of the lane in v-s-space. 

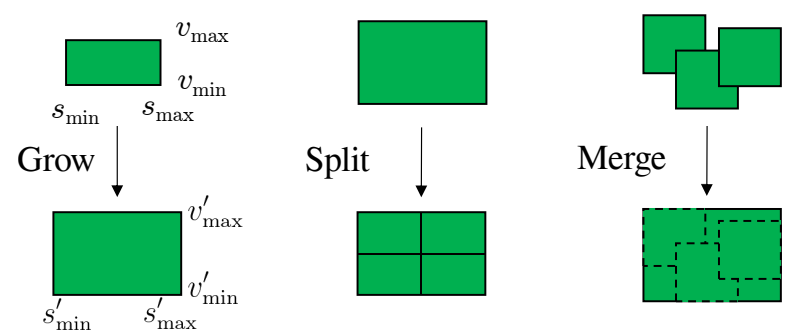

Fig. 3: Grow, split and merge operations for subsets.

\section{B. Operations on subsets}

We first define three geometric operations for a subset $\mathbf{S}$ : Grow, split and merge.

1) Grow: After a certain time period $t$, one subset $\mathbf{S}$ changes its $v_{\min }, v_{\max }, s_{\min }$ and $s_{\max }$. As the subset represents state intervals of a vehicle, the vehicle dynamics should be followed, i.e. subsets should grow following one acceleration interval $[\bar{a}, \underline{a}]$, with $\bar{a}, \underline{a} \in\left[a_{\min }, a_{\max }\right]$, where $a_{\min }$ and $a_{\max }$ represent the minimum and maximum acceleration of a vehicle.

After $t$, the new limits for velocity and longitudinal position of a subset can be represented by $v_{\min }^{\prime}, v_{\max }^{\prime}, s_{\min }^{\prime}$ and $s_{\max }^{\prime}$. One example is shown in Fig. 3. The new limits are computed following:

$$
\begin{gathered}
v_{\max }^{\prime}= \begin{cases}v_{\max }+t \bar{a} & \left(v_{\max }+t \bar{a} \leq v_{\text {limit }}\right) \\
v_{\text {limit }} & \left(v_{\max }+t \bar{a}>v_{\text {limit }}\right)\end{cases} \\
v_{\min }^{\prime}= \begin{cases}v_{\min }+t \underline{a} & \left(v_{\min }+t \underline{a} \geq 0\right) \\
0 & \left(v_{\min }+t \underline{a}<0\right)\end{cases} \\
s_{\max }^{\prime}= \begin{cases}s_{\max }+v_{\max } t+\frac{1}{2} \bar{a} t^{2} & \left(v_{\max }+t \bar{a} \leq v_{\text {limit }}\right) \\
v_{\text {limit }} t-\frac{\left(v_{\text {limit }}-v_{\max }\right)^{2}}{2 \bar{a}} & \left(v_{\max }+t \bar{a}>v_{\text {limit }}\right)\end{cases} \\
s_{\min }^{\prime}= \begin{cases}s_{\min }+v_{\min } t+\frac{1}{2} \underline{a} t^{2} & \left(v_{\min }+t \underline{a} \geq 0\right) \\
s_{\min }-\frac{v_{\min }^{2}}{2 \underline{a}} & \left(v_{\min }+t \underline{a}<0\right)\end{cases}
\end{gathered}
$$

2) Split: One subset can always be replaced by several subsets if the union of the latter can cover the same intervals for velocity and longitudinal position as the former. Therefore, one subset can be split into several subsets by dividing its velocity interval and the interval of the longitudinal position. One example is shown in Fig. 3.

3) Merge: The other way around, several subsets can also be replaced by one subset, if they can cover the same state intervals. However, as only four characteristic values $v_{\min }, v_{\max }, s_{\min }$ and $s_{\max }$ are defined for subsets, they can only be rectangular in v-s-space. Thus, for merging several subsets, we over-approximate one subset that picks the maximum $v_{\max }, s_{\max }$, and minimum $v_{\min }, s_{\min }$ of all subsets, as illustrated in Fig. 3.

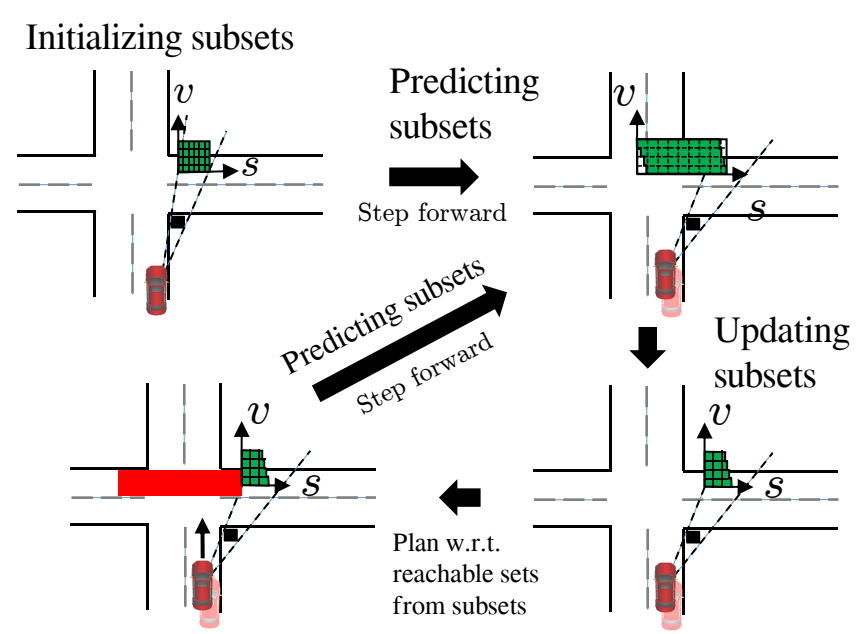

Fig. 4: The pipeline of tracking subsets and planning behaviors on top of the subsets. Green rectangles represent the subsets. The red polygon represents the predicted occupancy from the remaining subsets.

\section{Tracking subsets in occlusions}

In the entire paper, we assume a simple visibility model, by assuming a $360^{\circ}$ range sensor with a certain viewing range mounted on top of the vehicle center. The light blue polygon in Fig. 1 represents the FoV of the ego vehicle.

The goal of this approach is to track possible hidden TPs in occlusions thereby loosening the worst-case assumption and using this information for behavior planning. The pipeline (shown in Fig. 4) is done starting with initializing subsets on newly observed occlusions. By predicting the subsets with the operations in Section III-B and updating them with new observations, they can be tracked in a closed loop. At each planning step, the updated subsets can be utilized to generate reachable set predictions for the behavior planning algorithm. The generic way of generating set-based prediction from initial state intervals is detailed in [3].

1) Initializing subsets: This step is shown in the upper left part of Fig. 4. At the time where the ego vehicle observes an occlusion on the interesting lane, one state set $\mathcal{S}=\{(s, v)\}$ with $s \in\left[s_{1}, s_{2}\right]$ and $v \in\left[0 \frac{\mathrm{m}}{\mathrm{s}}, v_{\text {limit }}\right]$ can be initialized in v-s-space. $\mathcal{S}$ is able to cover all the possible states of potential vehicles in the initial occlusions. Then we select two discretization sizes $\Delta v$ and $\Delta s$ for velocity and longitudinal position, and split the initial set $\mathcal{S}$ into subsets, each of which covers one portion of possible states in occlusions.

2) Predicting subsets: After one time step, the ego vehicle moves to a slightly different position, and can therefore see different parts of the lane. Meanwhile, all the initialized subsets also change their characteristic values $v_{\min }, v_{\max }$, $s_{\min }$ and $s_{\max }$. The prediction step is explained in Fig. 5. The initialized subsets in the upper left part of Fig. 4 is again illustrated in the upper left of Fig. 5. Each subset has an initial size of $\Delta v$ and $\Delta s$. As mentioned before, all the subsets should grow according to an acceleration interval $\left[a_{\min }, a_{\max }\right]$ that corresponds to the acceleration limits 

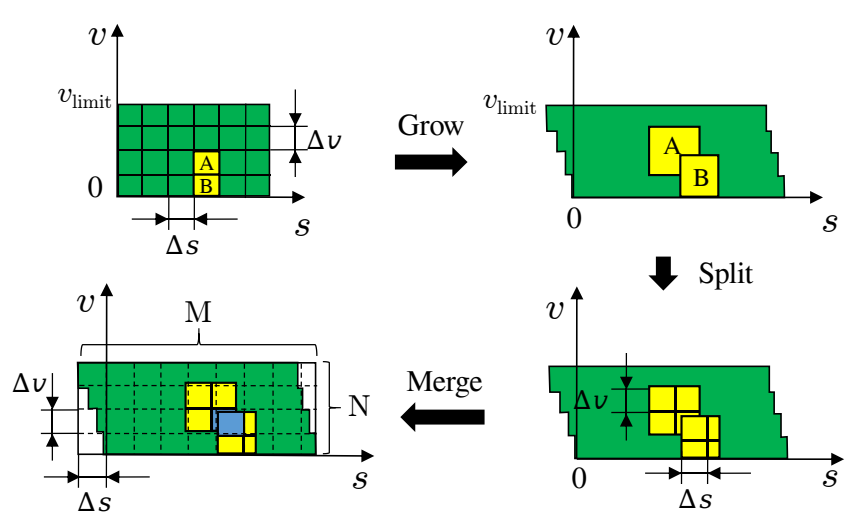

Fig. 5: Predicting subsets.

of the vehicles. As each subset has different characteristic values, they won't grow with the same speed and scalar. For instance, the subsets A and B in Fig. 5 will have different size according to (1) to (4) after growing. Large overlapping areas exist between each subset, and the union of them will not be a rectangle anymore as the upper right part of Fig. 5 depicts. The reason for this distorted shape is that the subsets in the upper part will move to the left faster than the subsets in the bottom, because they have an overall higher velocity.

Now after growing, the number of the subsets maintains, but they have different sizes, overlap with each other, and are highly disordered. We apply the split operation for all the subsets again with the discretization size of $\Delta v$ and $\Delta s$. For example, A and B will then be split into four subsets each. By that, the total number of subsets will explode exponentially. In order to avoid memory issue and keep computational pressure low, we limit the number of subsets by merging the subsets after splitting.

Although the union of all the split subsets will not be rectangular anymore, its outer contour can form a rectangle, as the left bottom part of Fig. 5 shows. We discretize the rectangular space again with $\Delta v$ and $\Delta s$, which formulates $\mathrm{M} \times \mathrm{N}$ grids in $\mathrm{v}$-s-space. Those split subsets will be merged into one, if their centers lie into the same grid, e.g. the two blue subsets in Fig. 5 will be merged into one, because their centers lie into the same grid formed by the dashed black lines. In the end, we obtain another set of subsets with a maximum number of $\mathrm{M} \times \mathrm{N}$, each of which has a maximum size of $2 \Delta v \times 2 \Delta s$, as shown in the upper right part of Fig. 4.

3) Updating subsets: As the ego vehicle already has a new FoV after one time step, the predicted subsets should be updated accordingly. We update subsets by either removing or keeping them. One subset will be removed, once any longitudinal position between its $s_{\min }$ and $s_{\max }$ is seen. As we choose $\Delta s$ much smaller than the length of a real vehicle, and the size of the subsets is controlled by the split-operation in prediction step, it is guaranteed that all the vehicles whose states covered by a subset can be seen, once any longitudinal position of a subset is visible. The updating step is depicted by the bottom right part of Fig. 4 .

The new subsets after updating can be used either for

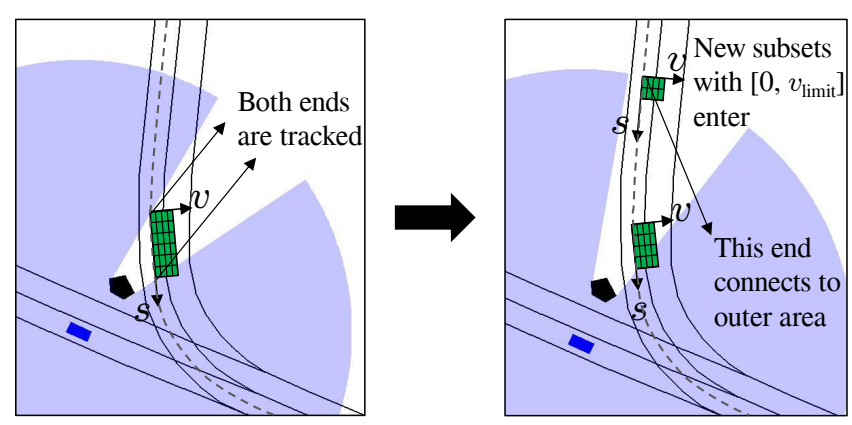

Fig. 6: Losing track on borders of occluded lane sections

planning the ego vehicle's behavior or for collision warning systems that assess the possible risk from occlusions.

\section{Losing track on borders of occluded lane sections}

Until now, we only considered the case where both ends of the occluded section of the lane are under our monitoring. Thus, we can make sure that no new vehicles from outside enter the occluded lane section, and the subsets that were initialized and tracked always build the limits for the potential hidden vehicles' states, as depicted by the green rectangles in the left figure of Fig. 6.

However, when one end of the occluded section extends beyond the FoV of the vehicle, as shown in the right figure of Fig. 6, the subsets alone can not cover all the possible states anymore. To overcome this problem, it is assumed that new subsets with velocity interval of $\left[0 \frac{\mathrm{m}}{\mathrm{s}}, v_{\text {limit }}\right]$ will enter the occlusion from the connecting point between the occluded section and the outer area, as soon as the connection is formed. The new subsets will be predicted and updated the same way as the existing subsets.

\section{EVALUATION}

The evaluation will be done in two parts. Firstly, the coverage of the subsets will be evaluated, and the ability that the subsets cover all the possible states in occlusions will be proved. Secondly, a proof-of-concept planning algorithm is implemented to present the better driving efficiency over baseline with our tracked subsets while providing the same safety as baseline.

In all the evaluations, the following parameters are used: $v_{\text {limit }}=13 \frac{\mathrm{m}}{\mathrm{s}}, \Delta v=1 \frac{\mathrm{m}}{\mathrm{s}}, \Delta s=0.2 \mathrm{~m}, a_{\max }=3 \frac{\mathrm{m}}{\mathrm{s}^{2}}$ and $a_{\min }=-4 \frac{\mathrm{m}}{\mathrm{s}^{2}}$. With this parameter setup and a simple Pythonimplementation, the tracking of one occluded lane-segment takes less than 30 milliseconds for each step on a laptop cpu (intel CORE i7 8th Gen).

\section{A. Coverage of the subsets}

In order to evaluate the coverage of the subsets, we initialize 1000 vehicles with random initial velocities and longitudinal positions in occlusions, at the same time we initialize our subsets in occlusion and start the tracking pipeline, as shown in the first row of Fig. 7. The randomly generated vehicles are assumed to move along the center of the lane. At each simulation step, they select one random 

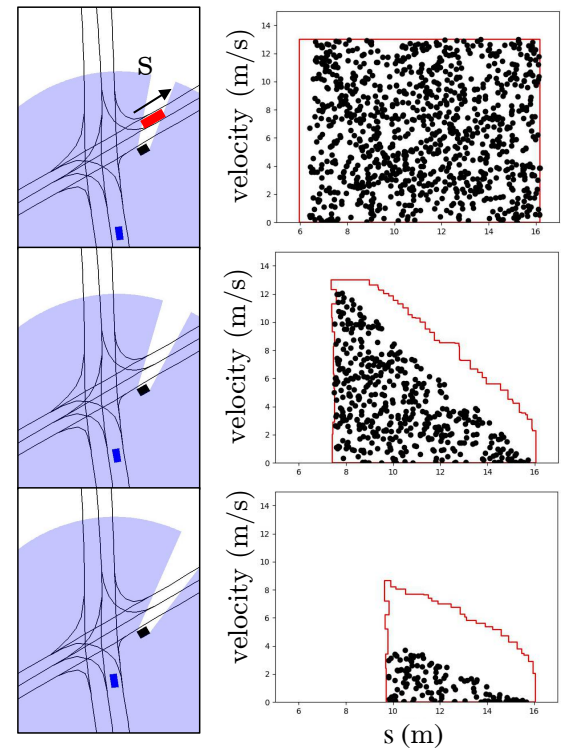

(a) $\mathrm{t}=0 \mathrm{~s}, 1 \mathrm{~s}, 2 \mathrm{~s}$
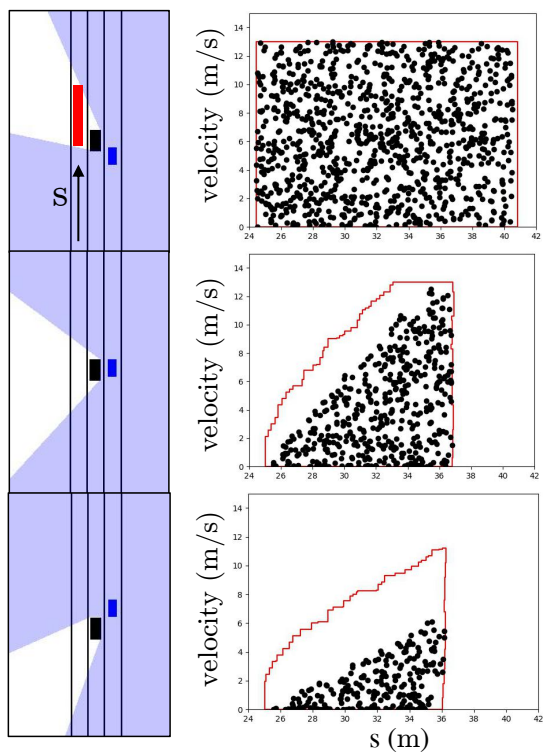

(b) $\mathrm{t}=0 \mathrm{~s}, 1.4 \mathrm{~s}, 2.8 \mathrm{~s}$
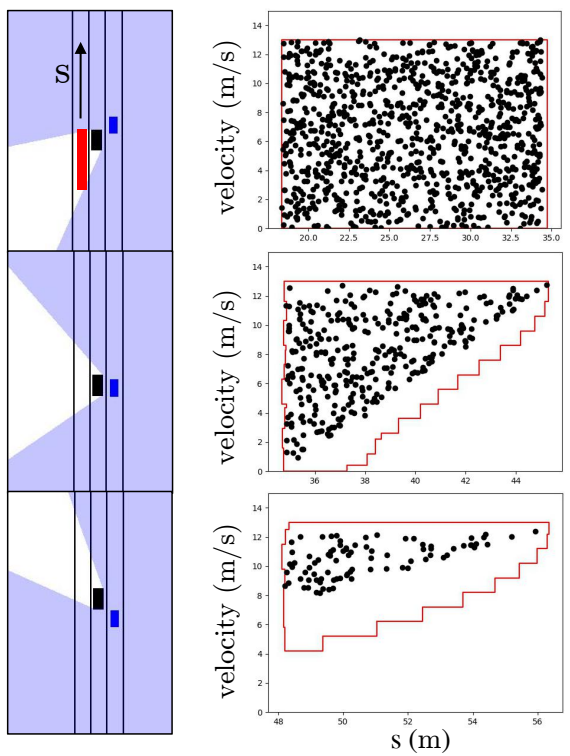

(c) $\mathrm{t}=0 \mathrm{~s}, 1.4 \mathrm{~s}, 2.8 \mathrm{~s}$

Fig. 7: Three scenarios where occlusions are tracked with subsets. The figures in the first row are the initial scenes, with the initial occluded lane sections represented by the red solid polygons. In the v-s-graphs on the right side, the hollow red polygons are the unions of the subsets. The black points represent the states of the remaining vehicles over time. The positive s-directions of the graphs are shown by the black arrows. (a) Ego vehicle (blue) approaches an unsignalized intersection. (b)(c) Ego vehicle (blue) and another vehicle (black) drive on a street with three lanes.

acceleration value between $a_{\min }$ and $a_{\max }$. The sampled vehicles will be removed as soon as they expose themselves to the ego vehicle's FoV. The states of the remaining vehicles in occlusions are shown by the black points, and the unions of the updated subsets are depicted by the red hollow polygons.

In Fig. 7(a), the ego vehicle approaches an unsignalized intersection. As it moves forward, the occlusion is pushed to the right side. Meanwhile, the number of the initialized hidden vehicles is decreasing, especially the ones with high velocities. At the same time, the high-speed part of the subsets is removed as well and the union of them tends to converge to fit the outer contour of the black points. In Fig. 7 (b), the ego vehicle with $10 \frac{\mathrm{m}}{\mathrm{s}}$ and another vehicle with $5 \frac{\mathrm{m}}{\mathrm{s}}$ drive on a street with three lanes. Similar to the scenario in Fig. 7(a), the high-speed part of the subsets and the sampled vehicles are reducing as the ego vehicle gains more information about the occlusion. In Fig. 7(c), on the same three-lane street, the ego vehicle drives with $5 \frac{\mathrm{m}}{\mathrm{s}}$ and the black vehicle drives with $10 \frac{\mathrm{m}}{\mathrm{s}}$. This time, the low-speed part of the subsets and the sampled vehicles are gradually removed. Otherwise, they can not hide behind the black vehicle that passes the ego vehicle with a higher velocity.

In all three scenarios, by tracking the subsets over time, they are able to cover the possible remaining vehicles that are simulated to move randomly. However, a gap at one edge of the subsets' union can be observed, as all the v-s-graphs in Fig. 7 show. One reason is that the sampled vehicles are moving with random acceleration, so the probability that they always move in an extreme way is low. Thus, they can not keep the subsets filled perfectly. The second reason is that, during our merge-step, a small over-approximation is introduced as well, which propagates forward and forms a larger gap over time. However, the merge-step is necessary for limit the number of subsets, and even with this small over-approximation, we can refine the state intervals in occlusions greatly compared to algorithms that always assume worst-case scenarios.

\section{B. Planning with subsets for gaining efficiency}

In this evaluation, the goal is to present that with the subsets that are tracked, a more efficient driving behavior can be achieved while reaching the same safety as in baseline.

The baseline algorithm that we compare to is from [3], i.e. vehicles are always possible to appear at the critical sensing edges of the occlusions with velocity interval of $\left[0 \frac{\mathrm{m}}{\mathrm{s}}, v_{\text {limit }}\right]$. In baseline, they account for reachable set prediction from their state intervals for ensuring safety. The same prediction can be employed to the subsets as well, as they are just special state intervals.

Although this approach can be applied to all types of occlusion, the evaluation focuses more on the scenarios where there is only a sliver of occlusion, e.g. caused by vegetation, poles or vehicles, rather than corners of walls where half the view is occluded. In the latter case, because only one end of the occlusion is tracked, the state interval obtained by our approach will be the same as the worst-case approach, as the new observation cannot really bring more information about velocities of the TPs in occlusion.

As for the proof-of-concept planner, the ego vehicle is assumed to move longitudinally along the ego lane. At each planning step, the planner selects one acceleration value from a set of acceleration candidates $\mathcal{A}=$ 


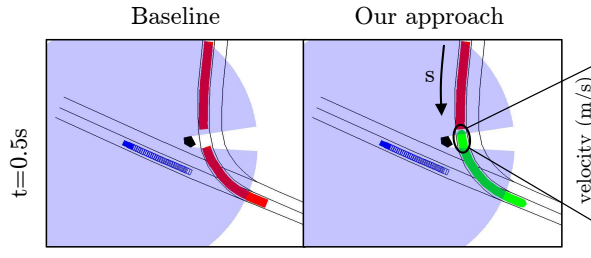

Subsets in occlusion
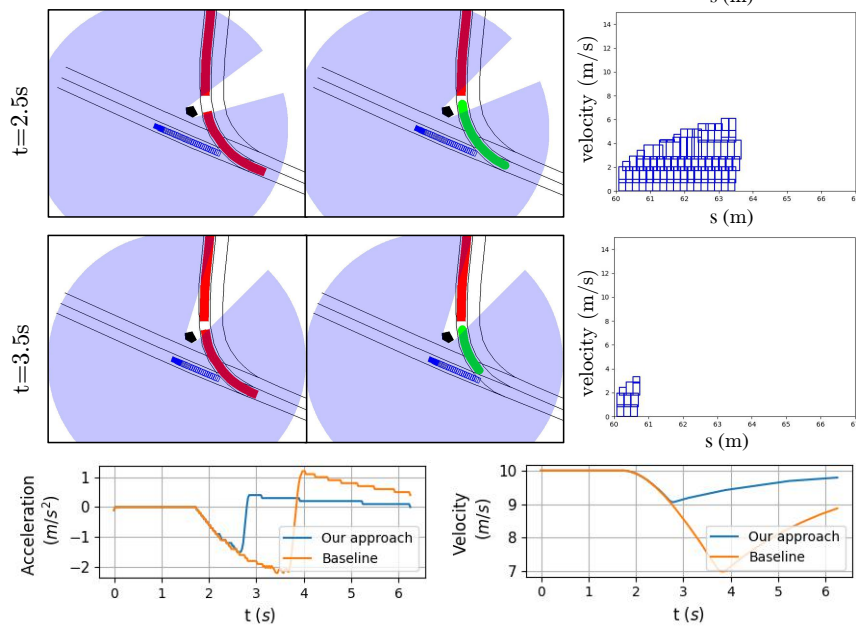

(a) Ego vehicle (blue) merges into main street
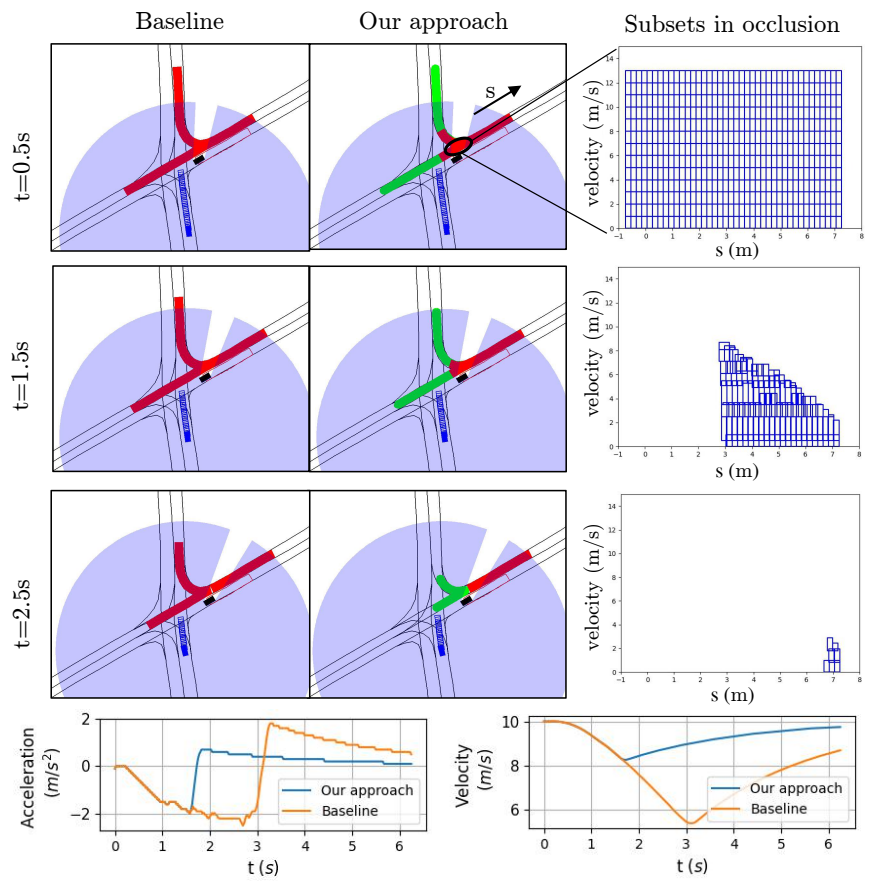

(b) Ego vehicle (blue) traverses an unsignalized intersection.

Fig. 8: Comparison between our approach and baseline with a proof-of-concept behavior planner. In both scenarios, the FoVs of the ego vehicle (blue) are occluded by the black obstacles. The first row shows the scenes where the subsets are initialized in the occlusions (black circles) at 0.5 seconds. The solid red polygons represent the set-based prediction from the potential vehicles in the worst case, and the solid green polygons represent the set-based prediction from the remaining subsets in tracked occlusions.

$\left\{-4 \frac{\mathrm{m}}{\mathrm{s}^{2}},-3.9 \frac{\mathrm{m}}{\mathrm{s}^{2}}, \ldots, 3 \frac{\mathrm{m}}{\mathrm{s}^{2}}\right\}$, and generates a trajectory that follows this constant acceleration. The planning horizon is chosen to be $3.5 \mathrm{~s}$ and the planning frequency is $25 \mathrm{~Hz}$. Those acceleration candidates, which end up with trajectories that intersect with the occupancy prediction of the state intervals in baseline or the subsets in our approach, are removed from $\mathcal{A}$ in the first place. From those remaining safe accelerations, the one which produces the lowest cost according to (5) will be the final decision.

$$
\operatorname{Cost}(a)=w_{1} a^{2}+w_{2}\left(1-k \frac{a}{a_{\max }}-\frac{v}{v_{d}}\right)^{2}
$$

where $a$ is the acceleration candidate and $v$ is the current velocity. $w_{1}, w_{2}$, and $k$ are parameters that should be tuned according to how the users balance utility and comfort. $v_{d}=$ $10 \frac{\mathrm{m}}{\mathrm{s}}$ represents the desired velocity of ego vehicle.

The comparison is done in two scenarios: 1. Merging into main street from one branch road with static occlusion (Fig. 8(a)). 2. Traversing an unsignalized intersection with dynamic occlusion caused by a moving vehicle (Fig. 8(b)). In both scenarios, the ego vehicle approaches with $10 \frac{\mathrm{m}}{\mathrm{s}}$.

In the first scenario, the ego vehicle decelerates because of the potential vehicles in occlusions and their occupancy predictions. However, with our approach, the subsets shrink as the ego vehicle steps forward (shown in the v-s-graphs of Fig. 8(a)), such that their occupancy predictions (shown by the green solid polygons in Fig. 8) are shorter compared to the ones from the baseline. It can be seen that as the ego vehicle moves, the subsets converge to an area that covers only the low-speed region. The human drivers would reason about the same after a period of observation because the vehicles should have exposed themselves already after a while if they would drive fast. In about 3.5 seconds, the subsets are almost eliminated and the ego vehicle is free to go. The acceleration and velocity profiles of the baseline and our approach are shown in the bottom graphs. In the second scenario, we have a dynamic occlusion, which is caused by a slow-moving vehicle on the right arm of the street (shown in black color). In this case, the subsets are eliminated even faster (in about 2.5 seconds).

In both scenarios, our approach needs less deleceration and achieves overall higher velocity while providing the same safety guarantee as the baseline.

\section{CONCLUSION AND FUTURE WORK}

We motivate our work with the purpose of reasoning about the bounds of possible TPs' states in occlusions in a systematic way, considering the useful historical information, instead of always assuming worst-case events or only estimating risk probabilistically according to the current scene. For this purpose, we introduce the state set and subsets, and a pipeline for tracking the subsets. To present the potential of our approach, it is first shown that all the possible states in occlusions can be covered by the subsets. By tracking of occluded areas we achieve a significantly less conservative driving behavior, while still guaranteeing safety under the minimal set of assumptions. This has been shown with a 
proof-of-concept planner in two scenarios with occlusions from static and dynamic obstacles.

In our approach, we only make assumptions about the vehicles having maximum and minimum accelerations and driving only forwards. Therefore, any type of TPs that fulfills these assumptions can be covered by the subsets. To further refine our approach, the assumption that the TPs only move along the lane with zero lateral offsets can be lifted, by integrating more dimensions to our subsets, e.g. lateral offset to the centerline and orientation, which makes the subsets propagate in three or four-dimensional space, the same tracking pipeline applies though.

Furthermore, after integrating those more dimensions into the subsets, it is also interesting to apply this approach in real traffic data in order to validate that all the TPs that exist in occlusion of the ego vehicle are really covered and tracked by the subsets. If any of them behaves beyond the capability of the subsets, analysing their behaviors is also benefitial for corner case evaluation as they exceed the preset parameter range for sure.

Finally, we would like to incorporate our approach with the FoV polygons that is generated by real range sensors, and evaluate the performance under real conditions.

\section{REFERENCES}

[1] S. Grigorescu, B. Trasnea, T. Cocias, and G. Macesanu, "A survey of deep learning techniques for autonomous driving," Journal of Field Robotics, vol. 37, no. 3, p. 362-386, Apr 2020. [Online]. Available: http://dx.doi.org/10.1002/rob.21918

[2] S. Shalev-Shwartz, S. Shammah, and A. Shashua, "On a formal model of safe and scalable self-driving cars," CoRR, vol. abs/1708.06374, 2017. [Online]. Available: http://arxiv.org/abs/1708.06374

[3] P. F. Orzechowski, A. Meyer, and M. Lauer, "Tackling occlusions \& limited sensor range with set-based safety verification," CoRR, vol. abs/1807.01262, 2018. [Online]. Available: http://arxiv.org/abs/1807.01262

[4] M. Althoff and S. Magdici, "Set-based prediction of traffic participants on arbitrary road networks," IEEE Transactions on Intelligent Vehicles, vol. 1, no. 2, pp. 187-202, June 2016.

[5] P. F. Orzechowski, K. Li, and M. Lauer, "Towards responsibilitysensitive safety of automated vehicles with reachable set analysis," in 2019 IEEE International Conference on Connected Vehicles and Expo (ICCVE), 2019, pp. 1-6.

[6] M. Naumann, H. Königshof, M. Lauer, and C. Stiller, "Safe but not overcautious motion planning under occlusions and limited sensor range," 2019.

[7] M. Yu, R. Vasudevan, and M. Johnson-Roberson, "Occlusionaware risk assessment for autonomous driving in urban environments," CoRR, vol. abs/1809.04629, 2018. [Online]. Available: http://arxiv.org/abs/1809.04629

[8] S. G. McGill, G. Rosman, T. Ort, A. Pierson, I. Gilitschenski, B. Araki, L. Fletcher, S. Karaman, D. Rus, and J. J. Leonard, "Probabilistic risk metrics for navigating occluded intersections," IEEE Robotics and Automation Letters, vol. 4, no. 4, pp. 4322-4329, 2019.

[9] F. Damerow, T. Puphal, Y. Li, and J. Eggert, "Risk-based driver assistance for approaching intersections of limited visibility," in 2017 IEEE International Conference on Vehicular Electronics and Safety (ICVES), 2017, pp. 178-184.

[10] C. Hubmann, N. Quetschlich, J. Schulz, J. Bernhard, D. Althoff, and C. Stiller, "A pomdp maneuver planner for occlusions in urban scenarios," 062019.

[11] X. Lin, J. Zhang, J. Shang, Y. Wang, H. Yu, and X. Zhang, "Decision making through occluded intersections for autonomous driving," in 2019 IEEE Intelligent Transportation Systems Conference (ITSC), 2019, pp. 2449-2455.

[12] D. Kamran, C. F. Lopez, M. Lauer, and C. Stiller, "Risk-aware highlevel decisions for automated driving at occluded intersections with reinforcement learning," 2020.
[13] L. Wang, C. F. Lopez, and C. Stiller, "Generating efficient behaviour with predictive visibility risk for scenarios with occlusions," in 2020 23 st International Conference on Intelligent Transportation Systems (ITSC), 2020, accepted.

[14] H. Andersen, W. Schwarting, F. Naser, Y. H. Eng, M. H. Ang, D. Rus, and J. Alonso-Mora, "Trajectory optimization for autonomous overtaking with visibility maximization," in 2017 IEEE 20th International Conference on Intelligent Transportation Systems (ITSC), Oct 2017, pp. $1-8$.

[15] F. Poggenhans, J.-H. Pauls, J. Janosovits, S. Orf, M. Naumann, F. Kuhnt, and M. Mayr, "Lanelet2: A high-definition map framework for the future of automated driving," 11 2018, pp. 1672-1679. 